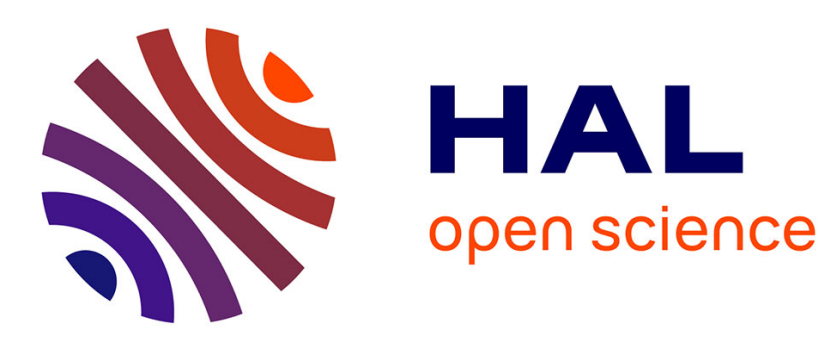

\title{
Electroactive mixed self-assembled monolayers: A numerical overview of phase segregations
}

\author{
Olivier Alévêque, Eric Levillain, Yohann Morille
}

\section{To cite this version:}

Olivier Alévêque, Eric Levillain, Yohann Morille. Electroactive mixed self-assembled monolayers: A numerical overview of phase segregations. Electrochemistry Communications, 2014, pp.17-22. 10.1016/j.elecom.2014.05.009 . hal-01153492

\section{HAL Id: hal-01153492 \\ https://hal.science/hal-01153492}

Submitted on 16 Jul 2020

HAL is a multi-disciplinary open access archive for the deposit and dissemination of scientific research documents, whether they are published or not. The documents may come from teaching and research institutions in France or abroad, or from public or private research centers.
L'archive ouverte pluridisciplinaire HAL, est destinée au dépôt et à la diffusion de documents scientifiques de niveau recherche, publiés ou non, émanant des établissements d'enseignement et de recherche français ou étrangers, des laboratoires publics ou privés. 


\section{Accepted Manuscript}

Electroactive mixed self-assembled monolayers: A numerical overview of phase segregations

Olivier Alévêque, Eric Levillain, Yohann Morille

PII:

DOI:

S1388-2481(14)00139-8

Reference: $\quad$ ELECOM 5152

To appear in: Electrochemistry Communications

Received date: $\quad 15$ April 2014

Revised date: $\quad 3$ May 2014

Accepted date: $\quad 6$ May 2014

Please cite this article as: Olivier Alévêque, Eric Levillain, Yohann Morille, Electroactive mixed self-assembled monolayers: A numerical overview of phase segregations, Electrochemistry Communications (2014), doi: 10.1016/j.elecom.2014.05.009

This is a PDF file of an unedited manuscript that has been accepted for publication. As a service to our customers we are providing this early version of the manuscript. The manuscript will undergo copyediting, typesetting, and review of the resulting proof before it is published in its final form. Please note that during the production process errors may be discovered which could affect the content, and all legal disclaimers that apply to the journal pertain. 
Electroactive mixed self-assembled monolayers: a numerical overview of phase segregations.

Olivier Alévêque, Eric Levillain*, Yohann Morille

Université d'Angers, CNRS, MOLTECH-Anjou UMR 6200, 2 bd Lavoisier, 49045 ANGERS cedex, France.

* Corresponding authors: Tel.: (+33)241735090; Fax: (+33)241735405; E-mail: eric.levillain@univ-angers.fr

\begin{tabular}{l|l}
\hline ARTICLE INFO & ABSTRACT \\
\hline $\begin{array}{l}\text { Keywords: } \\
\text { Self-assembled monolayers } \\
\text { Lateral interactions } \\
\begin{array}{l}\text { Phase segregation } \\
\text { Beta distribution }\end{array}\end{array}$ & $\begin{array}{l}\text { We propose a modelling of phase segregations, inspired by image filtering, } \\
\text { and dedicated to electrochemical systems. This original approach is } \\
\text { compared to previous experimental results and enhanced with the } \\
\text { concepts of 2D segregation and site percolation threshold. }\end{array}$ \\
\hline
\end{tabular}

\section{Introduction}

Since their discovery in 1946 [1,2], self-assembled monolayers (SAMs) have become an ideal system for the theoretical study of interfacial phenomena [3]. In the field of electrochemistry, there is clear evidence that electrochemical interfacial reactivity depends on the $2 \mathrm{D}$ distribution of electroactive sites adsorbed on a conducting solid surface.

Random distribution and phase segregation have been discussed in literature but very few papers have proposed a modelling of non-random distributions. In electrochemistry, the electrostatic model of Smith and White [4], then refined by Yoneyama et al. [5] with inclusion of the potential-independent by ion pairing and triple-ion formation, provided an analytical expression for the interfacial potential distribution, predicting the current-voltage shape, explaining a broadening and shifts of formal potentials of voltammetric waves (CVs). However, electrostatic approaches, involving intricate mathematical treatments and only dedicated to random distribution, are difficult to use. Without an "as clear" physical approach, the lateral interaction model [6] has been extended to any spatial distribution by introducing the statistical parameter $\phi$, that quantifies the local intersite interactions [7]. For a given distribution, this model enabled current-voltage behaviors to be simulated and allowed extracting characteristics parameters $\left(E_{p}, i_{p}\right.$ and FWHM) of CVs. Unfortunately, because of its local nature, the parameter $\phi$ do not allow to characterize the phase segregation as a whole. Here, we propose an original approach of phase segregations inspired by image filtering and we attempt to describe the concept of phase segregation by indicators from various scientific fields.

\section{Extended lateral interactions model}

To summarize previous works, the generalized lateral interactions model is defined according to the main following hypotheses $[6,7,8,9,10]$ :

- The electroactive centers are distributed on substrate with a unimodal statistical distribution. A parameter $\phi(\theta)$, between 0 and 1 , defined for a normalized surface coverage $\theta$, quantifies the 
nearest redox neighbors of the electroactive centers. For a random distributed SAM, $\phi(\theta)=\theta$, and when a phase segregation exist on the surface, $\phi(\theta)>\theta$.

- The sum of normalized surface coverage $\theta_{\circ}$ and $\theta_{R}$ of oxidized $(O)$ and reduced $(R)$ species is constant and equal to $\theta$,

- The surface occupied by $O$ is equal to the surface occupied by $R$,

- The electrochemical rate constant $k_{s}$ is independent of the coverage,

- $a_{O O}, a_{R R}$ and $a_{O R}$ are the interaction constants between molecules of $O$ and $R$. $a_{i j}$, independent of the potential and distribution, is positive for an attraction and negative for a repulsion.

For $\mathrm{k}_{\mathrm{s}} \rightarrow+\infty$, CVs are fully reversible and the parameters as full width at half maximum (FWHM), peak potential $\left(E_{p}\right)$ and peak current $\left(i_{p}\right)$ are defined as:

$\mathrm{E}_{\mathrm{p}}(\phi(\theta))=\mathrm{E}_{0}^{\prime}+\frac{\mathrm{RT}}{\mathrm{nF}} \mathrm{S} \phi(\theta)$

$\mathrm{i}_{\mathrm{p}}(\phi(\theta), \theta)=\frac{\mathrm{n}^{2} \mathrm{~F}^{2} v A \Gamma_{\mathrm{m}}}{\mathrm{RT}} \frac{\theta}{2(2-\mathrm{G} \phi(\theta))}$

$\mathrm{FWHM}(\phi(\theta)) \stackrel{|G \phi(\theta)<1|}{\approx} \frac{\mathrm{RT}}{\mathrm{nF}}\left(2 \ln (2 \sqrt{2}+3)-\frac{3 \sqrt{2}}{2} \mathrm{G} \phi(\theta)\right)$

with $\mathrm{G}=\mathrm{a}_{\mathrm{OO}}+\mathrm{a}_{\mathrm{RR}}-2 \mathrm{a}_{\mathrm{OR}}$ and $\mathrm{S}=\mathrm{a}_{\mathrm{RR}}-\mathrm{a}_{\mathrm{OO}} \quad(|\mathrm{G}|$ and $|\mathrm{S}| \leq 2)$

\section{Numerical models}

A mixed SAM can numerically be approached by an n-by-n matrix (M) composed of pixels: a pixel "1" (P1) for a site occupied by a redox species and a pixel "0" (P0) for a non-electroactive species (i.e. a diluent). The ratio between the number of $\mathrm{P} 1\left(\mathrm{~N}_{\mathrm{P} 1}\right)$ and the dimension of the matrix (i.e. $\left.\mathrm{n}^{2}\right)$ corresponds to the normalized surface coverage of electroactive species $\theta$ (i.e. $\theta=\frac{N_{P 1}}{n^{2}}$ ) [10].

\subsection{Generated numeric distributions}

To compute a set of images with different $2 \mathrm{D}$ distributions, we performed three operations.

First, an n-by-n matrix $\left(\mathrm{M}_{\mathrm{RND}}\right)$ of pseudorandom uniform values is generated on the open interval $(0,1)$.

Second, the matrix MRND is convoluted once or several times with a 2-D digital filter [i.e. a median filter, a square or circular averaging filter (later called disk), a rotationally symmetric Gaussian low pass filter, a Savitzky-Golay filter... etc.] in order to generate an $n$-by-n filtered matrix $\left(\mathrm{M}_{\text {Filtered }}\right)$ of smoothing values on the open interval $(0,1)$ and centered to 0.5 .

Last, a set of binary matrix $M_{\theta}$ is extracted from the filtered matrix by a basic binary thresholding, expressed as:

$M_{\theta}(i, j)= \begin{cases}1 & \text { if } M_{\text {Filtered }}(i, j)>\text { THRESHOLD } \\ 0 & \text { otherwise }\end{cases}$

Note that: 
- THRESHOLD parameter varies on the open interval $(0,1)$,

- A given threshold generates a binary matrix at a given P1/P0 ratio (i.e. a normalized surface coverage $\theta$ ),

- Threshold $=\theta$ only for a random matrix $\mathrm{M}_{\mathrm{RND}}$,

- All calculations, performed with MATLAB ${ }^{\circledR}$, are stable, convergent and quasi-independent of 2-D filter,

- The accuracy of results increases with the size of matrix and is high from $n=1000$.

As shown on figure 1, a succession of two-dimensional normalized convolution increases the smoothing effect and the images suggest a phase segregation. Note that the fractal dimension, estimated from boxcounting method, of binary images does not depend on the number of convolutions for a given P1/P0 ratio, which means that the smoothing effect preserves the random character of binary matrix whatever the scale.

\subsection{Estimation of $\phi(\theta)$ parameter}

A dimensionless quantity $\phi$, representative of the average number of lateral interactions per electroactive site is estimated by counting, for each P1 pixel, the P1 nearest neighbors, expressed as:

$$
\phi(\theta)=\frac{1}{K_{\text {Lattice }}} \cdot \frac{\sum_{i j}\left[M_{\theta}(i, j) \times N_{i j}\right]}{\sum_{i j}\left[M_{\theta}(i, j)\right]} \text { with }\left\{\begin{array}{l}
M_{\theta}=\text { binary matrix for a given } \mathrm{P} 1 / P 0 \text { ratio } \\
N_{i j}=\text { number of nearest neighbours } \\
K_{L \text { Lattice }}=8
\end{array}\right.
$$

Figure 2A represents variations of $\phi$ parameter vs. $\theta$ for successive two-dimensional normalized convolutions.

We can notice that the variations of $\phi$ vs. $\theta$ cover the whole area where a phase segregation is expected (i.e. $\phi>\theta)$. As a reminder, the $\phi<\theta$ area is inaccessible because there is nothing more random than a random distribution.

In addition, two successive convolutions with a 3-by-3 disk filter agree with the CNT distribution [7,10] and with experimental data [7] (Figure 2B).

Based on a bayesian approach which assumes that unknown parameters (i.e. smoothing effect) come from known distributions (i.e. random distribution), $\phi$ vs. $\theta$ could be approximated to the probability cumulative density function of the beta distribution (BetaCDF), for $0 \leq \theta \leq 1$, expressed as:

$\phi(\theta)=\frac{\int_{0}^{\theta} t^{\alpha-1} \cdot(1-t)^{\beta-1} d t}{\int_{0}^{1} t^{\alpha-1} \cdot(1-t)^{\beta-1} d t}$

Figure $2 \mathrm{C}$ provides evidence of a good agreement between numerical and mathematical approaches. There is no clear physical or statistical interpretation of $\alpha$ and $\beta$ parameters. Mathematically, $\alpha$ varies between the two boundaries (i.e. 0 for a full segregation and 1 for a random distribution). The radius of curvature mainly depends on $\alpha$, reflecting the strength of the phase segregation (Figure 2D). By contrast, $\beta$ modifies the curvature of the asymptote at $\theta=1$. 


\subsection{Connected-component labelling approach}

To evaluate the behavior of clusters, we used the well-known connected-component labelling, an algorithmic application of graph theory dedicated to detect connected regions in binary digital images. This algorithm traverses the binary matrix, labelling the vertices based on the connectivity (4 or 8-connected) and relative values of their neighbors.

For each binary matrix on a 4-connected connectivity, the numbers of clusters $\left(\mathrm{N}_{\mathrm{C}}\right)$ has been estimated versus $\theta$. Note that a high number of clusters implies that their size is small and thus the number of clusters of a random distribution will always be higher than the one of a phase segregation, for a same value of $\theta$.

$N_{C}$ vs. $\theta$ reaches to a maximum because the lower and upper limits are $N_{C}(\theta \rightarrow 0)=0$ and $N_{C}(\theta=1)=1$ respectively and successive convolutions increase the size of clusters, while decreasing their number. The relative variation of number of clusters $\Delta N_{C}$ (i.e. $\left.\Delta N_{C}=\frac{\left(N_{P 1}-N_{C}\right)}{N_{P 1}}\right)$ versus $\theta$ quantifies the clusters growth (Figure 3A). As expected, $\Delta N_{C}$ vs. $\theta$ is a monotonic increasing curve in the semi-open interval $(0,1)$, increasing with successive convolutions.

In keeping with the Johnson-Mehl-Avrami-Kolmogorov formalism [11], dedicated to describe how solids transform from one phase (state of matter) to another at constant temperature, and the classical nucleation theory, $\Delta \mathrm{N}_{C}$ vs. $\theta$ could be approximated to a transformation "kinetic" (i.e. $t \rightarrow \frac{\theta}{(1-\theta)}$ ) of one phase (P0) from another (P1) by the growth of clusters and modelled by an Avrami equation [12], expressed as:

$\Delta \mathrm{N}_{\mathrm{C}}=1-\exp \left[-\mathrm{K}\left(\frac{\theta}{1-\theta}\right)^{\mathrm{n}}\right]$

Figure 3B shows a good agreement between numerical data and equation 7 . There is no clear physical interpretation of the Avrami constants $K$ and $n$ but it is recognized that $n$ reflects the nature of the transformation (Figure 3C).

As the connected-component labelling algorithm computes the numbers and the size of clusters of binary matrix, the variation of the largest clusters normalized to $N_{P 1}$ versus $\theta$ allows estimating the site percolation threshold (i.e. the $\theta$ value from which all pixels $\mathrm{P} 1$ are interconnected) for a given number of loops (Figure 3D). The threshold of random distribution agrees with the one of a square lattice (i.e. 0.593) [13] then it decreases with successive convolutions to reach a lower limit (i.e. 0.50).

Beyond the threshold, all pixels P1 form only one phase whatever the distribution, which can play a crucial role on the charge transport on SAMs [14].

\subsection{D segregation}

Attempting to develop a physical model is premature without a clear interpretation because the phase segregation is a large and complicated issue, governed, amongst others, by the interplay of coulombic repulsion, pairing ions and solvatation (for instance in electrochemical field, see reference [15]). Moreover, it is well-known that Ising spin models in physical field and random Markow fields (close to the Schelling model) in mathematical field involve very intricate mathematical treatments without analytical solution [16]. 
A solution, which has its origins in the information field, could be a statistical approach of the 2D segregation (S) according to the entropy $(H)$ defined by Shannon [17], then refined by Kapur and Kesavan [16], expressed as:

$$
\begin{gathered}
\mathrm{S}(\theta)=1-\mathrm{H}(\theta)=1-\frac{-1}{\mathrm{n}^{2} \ln (2)} \sum_{\mathrm{ij}}\left[\frac{1+\mathrm{N}_{\mathrm{ij}}}{1+\mathrm{K}_{\text {Lattice }}} \ln \left(\frac{1+\mathrm{N}_{\mathrm{ij}}}{1+\mathrm{K}_{\text {Lattice }}}\right)+\frac{\mathrm{K}_{\text {Lattice }}-1}{1+\mathrm{K}_{\text {Lattice }}} \ln \left(\frac{\mathrm{K}_{\text {Lattice }}-\mathrm{N}_{\mathrm{ij}}}{1+\mathrm{K}_{\text {Lattice }}}\right)\right] \\
\text { with }\left\{\begin{array}{l}
\mathrm{N}_{\mathrm{ij}}=\text { number of nearest neighbours of } \mathrm{P} 0 \text { and } \mathrm{P} 1 \\
\mathrm{~S}=0 \Rightarrow \text { perfect blend of } \mathrm{P} 0 \text { and } \mathrm{P} 1 \text { (i.e. a chessboard) } \\
\mathrm{S}=1 \Rightarrow \text { full segregation }
\end{array}\right.
\end{gathered}
$$

As expected, $S(0)$ and $S(1)$ are equal to 1 and $S$ vs. $\theta$ reaches to a minimum value at $\theta=0.5$, which increases with successive convolutions to converge towards $S=1$ (Figure $4 \mathrm{~A}$ ). Because equation 8 takes into account the 2D segregation of all pixels (i.e. P0 and P1) and because the successive convolutions preserve the random character of binary matrix, $S$ vs. $\theta$ is a symmetric curve. It is notable that the shape of $S$ vs. $\theta$, that can be approximated by a probability density function of the beta distribution (Figure 4B), is independent of successive convolutions (Figure $4 \mathrm{C}$ and $4 \mathrm{D}$ ). Should it be related to the independence of the fractal dimension with the convolution?

Contrary to generally accepted ideas, this entropic approach suggests that the $2 \mathrm{D}$ segregation $(S>0)$ exists whatever the distribution, even to a random distribution therefore.

\section{Conclusion}

Based on this work, it appears possible to link the notion of local intersite interactions ( $\phi$ vs. $\theta$ and, to date, only reachable by electrochemistry) to the concept of phase segregation ( $S$ vs. $\theta$ ) and thus, to estimate, via abacus (figures $2 \mathrm{~A}$ and $4 \mathrm{~A}$ respectively), the percentage of $2 \mathrm{D}$ segregation of a mixed SAM from electrochemical data.

The connected-component labelling associated to the valuation of the site threshold percolation should enable to better understand the mechanisms of charge-transport on SAMs.

We hope that this approach will provide tools to assist in establishing detailed structure-reactivity relationships for interfacial reactions on SAMs, especially on mixed SAMs.

\section{Acknowledgments}

All calculations were performed with $\mathrm{MATLAB}^{\circledR}$ and $\mathrm{SCILAB}^{\odot}$.

\section{References}

1 W. C. Bigelow, D. L. Pickett, W. A. Zisman, Films adsorbed from solution in nonpolar liquids, Journal of Colloid Interface Science, 1 (1946), 513-538.

2 R.G. Nuzzo, D.L. Allara, Adsorption of bifunctional organic disulfides on gold surfaces, Journal of the American Chemical Society, 105 (1983) 4481-4483.

3 J.C. Love, L.A. Estroff, J.K. Kriebel, R.G. Nuzzo, G.M. Whitesides, Self-assembled monolayers of thiolates on metals as a form of nanotechnology, Chem. Rev., 105 (2005) 1103-1169. 
4 C. P.Smith, H. S. White, Voltammetric Response of Electrodes Coated with Electroactive Molecular Films, Analytical Chemistry, 64 (1992) 2398-2405.

5 M. Ohtani, S. Kuwabata, H. Yoneyama, Voltammetric Response Accompanied by Inclusion of Ion Pairs and Triple Ion Formation of Electrodes Coated with an Electroactive Monolayer Film, Analytical Chemistry 69 (1997) 1045-1053.

6 E. Laviron, Surface linear potential sweep voltammetry: Equation of the peaks for a reversible reaction when interactions between the adsorbed molecules are taken into account, Journal of Electroanalytical Chemistry and Interfacial Electrochemistry, 52 (1974) 395-402.

7 O. Aleveque, P.Y. Blanchard, C. Gautier, M. Dias, T. Breton, E. Levillain, Electroactive self-assembled monolayers: Laviron's interaction model extended to non-random distribution of redox centers, Electrochemistry Communications, 12 (2010) 1462-1466.

8 E. Laviron, General expression of the linear potential sweep voltammogram in the case of diffusionless electrochemical systems, Journal of Electroanalytical Chemistry and Interfacial Electrochemistry, 101 (1979) 19-28.

9 E. Laviron, L. Roullier, General expression of the linear potential sweep voltammogram for a surface redox reaction with interactions between the adsorbed molecules: Applications to modified electrodes, Journal of Electroanalytical Chemistry and Interfacial Electrochemistry, 115 (1980) 65-74.

10 O. Aleveque, C. Gautier, M. Dias, T. Breton, E. Levillain, Phase segregation on electroactive selfassembled monolayers: a numerical approach for describing lateral interactions between redox centers, Physical Chemistry Chemical Physics, 12 (2010) 12584-12590.

11 W. Johnson, R. Mehl, Reaction kinetics in processes of nucleation and growth. Transactions of the Metallurgical Society of AIME, 135 (1939) 416-458.

12 M. Avrami, Kinetics of Phase Change. I. General Theory. Journal of Chemical Physics 7 (12): 11031112.

13 Z V. Djordjevic, H. E. Stanley, A. Margolina, Site percolation threshold for honeycomb and square lattices. Journal of Physics A: Math. Gen. 15 (1982) L405-L412.

14 S. G. J. Mathijssen, E. C. P. Smits, Paul A. van Hal, H. J. Wondergem, S. A. Ponomarenko, A. Moser, Roland Resel, P. A. Bobbert, M. Kemerink, R. A. J. Janssen, D. M. de Leeuw. Monolayer coverage and channel length set the mobility in self-assembled monolayer field-effect transistors. Nature Nanotechnology 4, 674 - 680 (2009).

15 C. Costentin, J.-M. Saveant, Dimerization of electrochemically generated ion radicals: mechanisms and reactivity factors. Journal Electroanalytical Chemistry 564 (2004) 99-113.

16 J. N. Kapur, H. K. Kesavan, Entropy Optimization Principles with Applications, Academic Press, San Diego, CA, 1992.

17 C. E. Shannon, A Mathematical Theory of Communication. Bell System Technical Journal, 27 (1948) 379-423 and 623-656. 


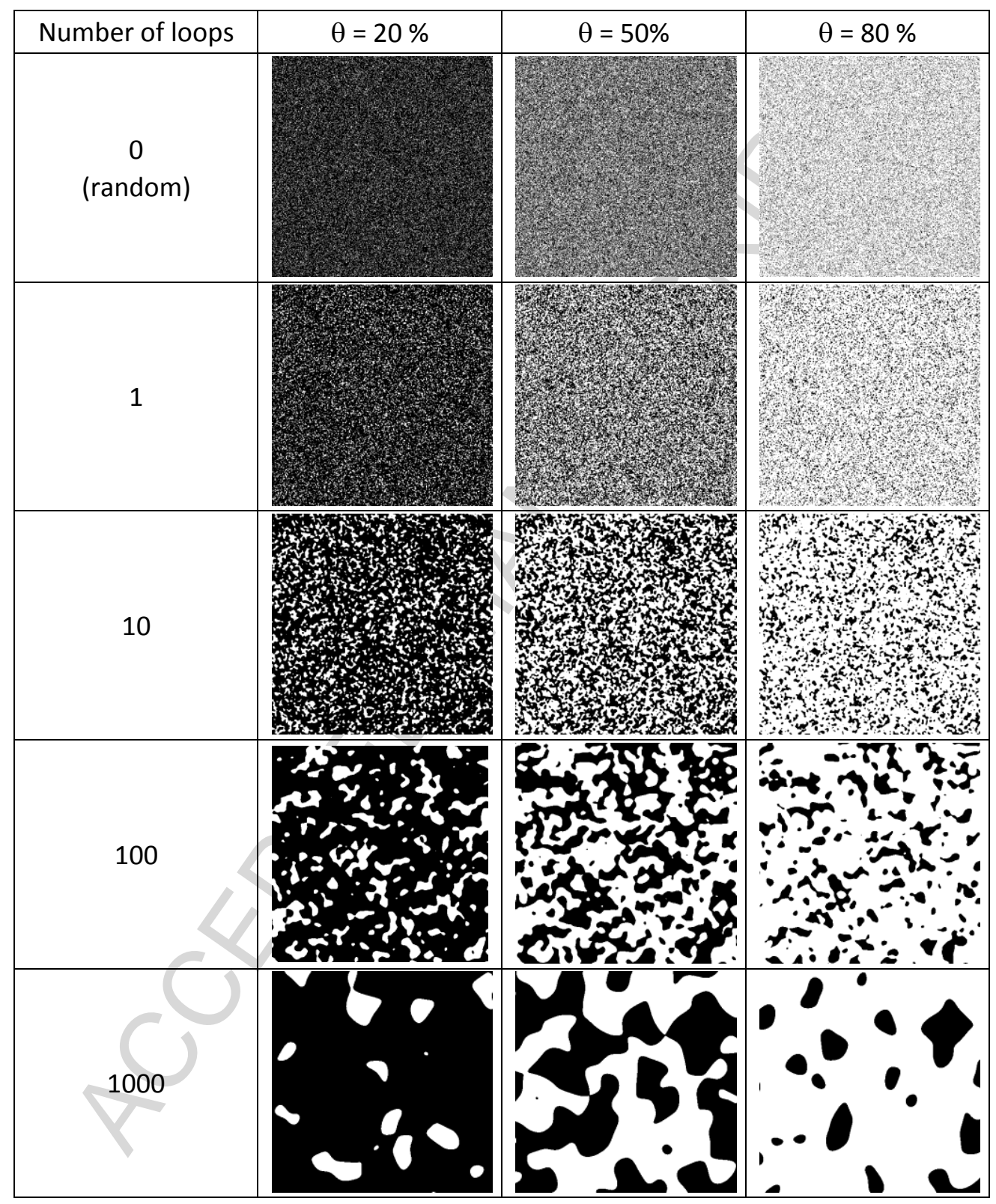

Figure 1: set of 5000-by-5000 binary matrix $(10 \times$ zoom - P0 $=$ black color and $\mathrm{P} 1=$ white color $)$ obtained with an 3-by-3 averaging filter (i.e. all elements equal to $1 / 9$ ) 
(A)

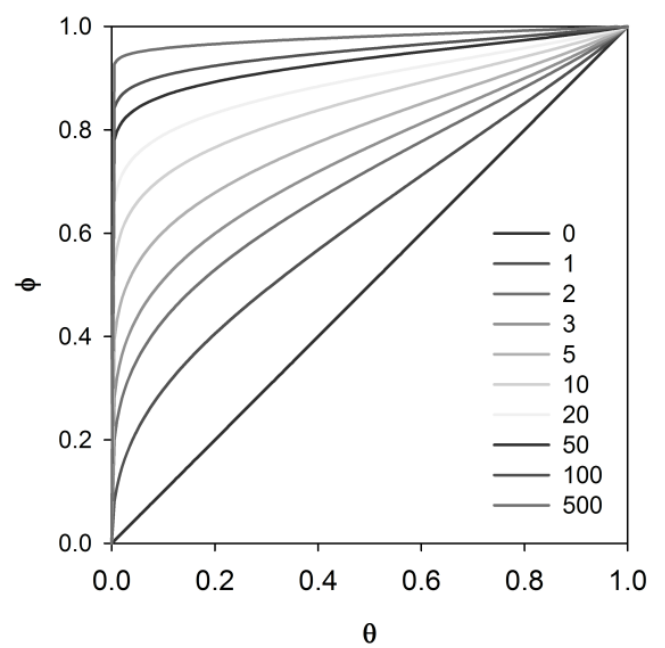

(C)

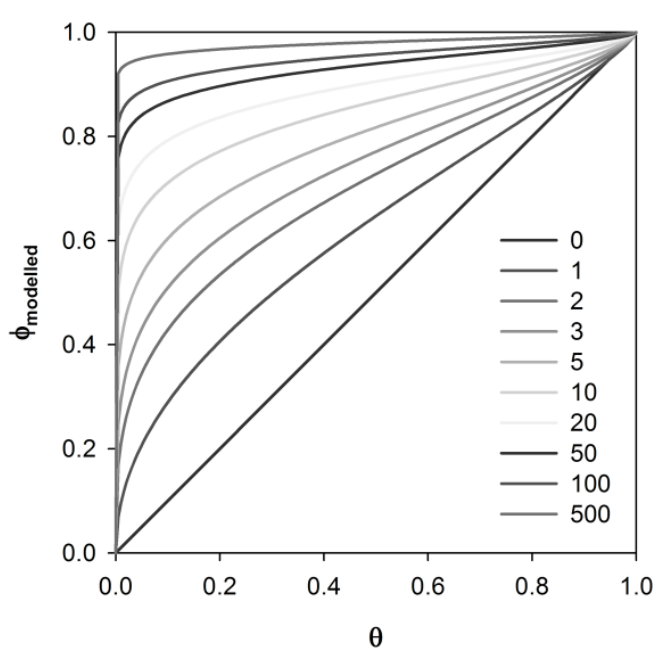

(B)

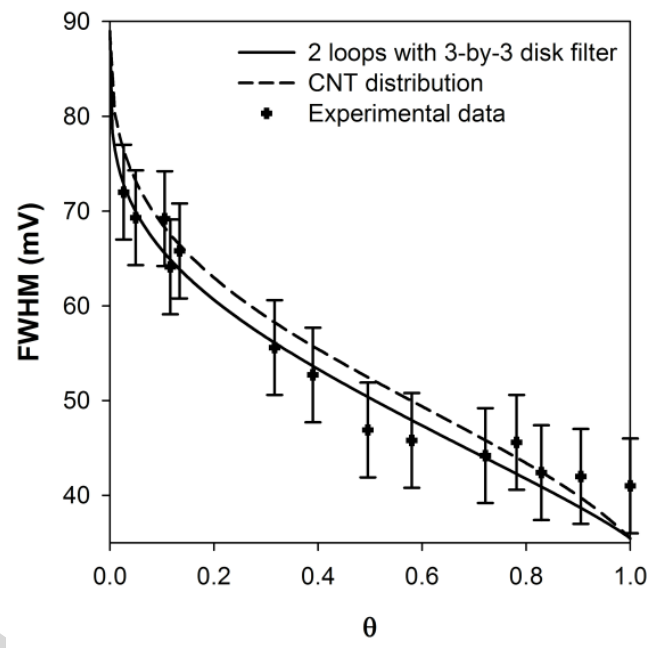

(D)

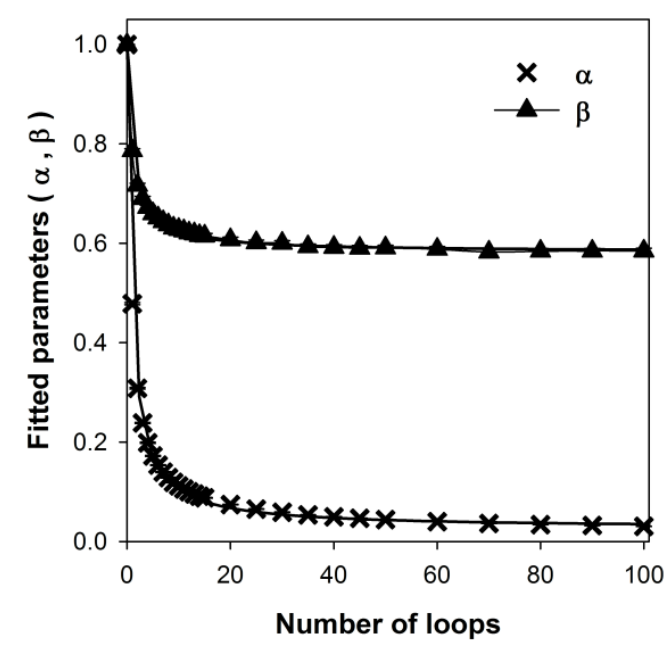

Figure 2:

(A) $\phi$ vs. $\theta$ extracted from successive two-dimensional normalised convolution (0 to 500 ) of random matrix and 3-by-3 2-D disk filter.

(B) FWHM vs. $\theta$ for CNT distribution, two successive convolution of random matrix and 3-by-3 2-D disk filter and experimental extracted from figure 4 of reference 6 (see equation 3 for the relationship between FWHM and $\phi)$.

(C) Using the Levenberg-Marquardt algorithm, $\phi$ was modelled by the probability cumulative density function of the beta distribution and the best fit was named $\phi_{\text {Modelled. }}$.

(D) $\alpha$ and $\beta$ parameters extracted from the fitting of $\phi$ vs. $\theta$ and BetaCDF function. 
(A)

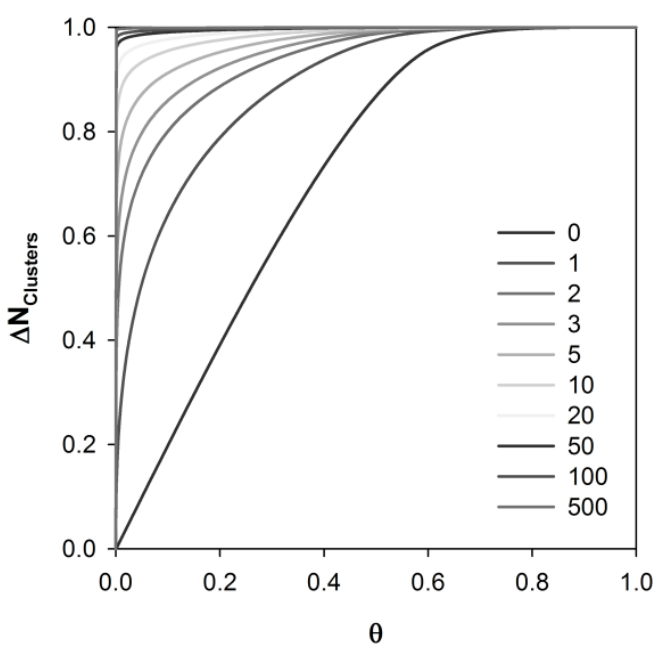

(C)

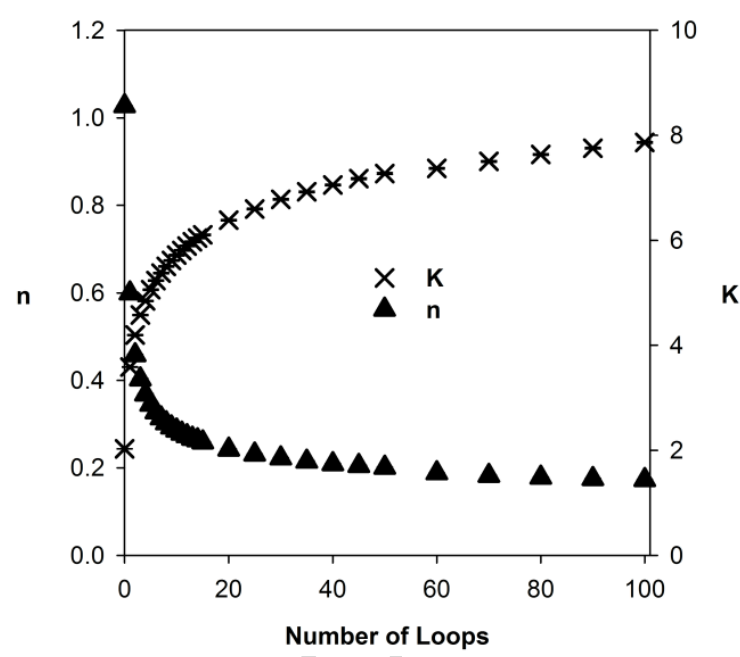

(B)

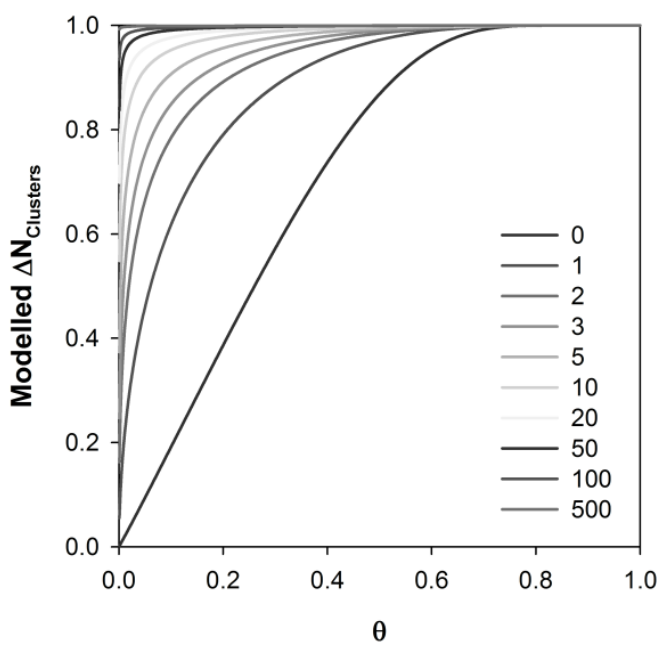

(D)

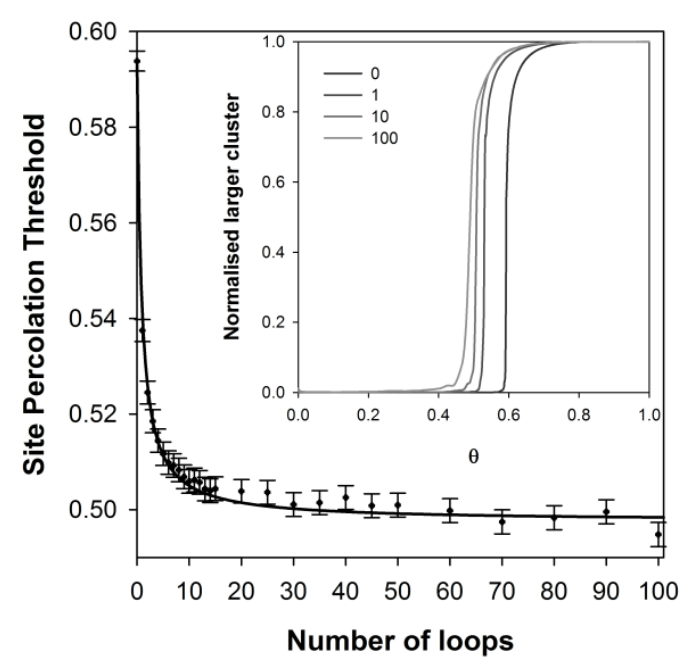

Figure 3:

(A) $\Delta \mathrm{N}_{\text {Clusters }}$ vs. $\theta$ extracted from successive two-dimensional normalised convolution ( 0 to 500 ) of random matrix and 2-D disk filter.

(B) Using the Levenberg-Marquardt algorithm, $\Delta \mathrm{N}_{\text {Clusters }}$ was modelled by the KJMA formalism and the best fit was named Modelled $\Delta \mathrm{N}_{\text {Clusters. }}$.

(C) $\mathrm{n}$ and $\mathrm{K}$ parameters extracted from the fitting of $\Delta \mathrm{N}_{\text {Clusters }}$ vs. $\theta$ and "Avrami" function.

(D) Site percolation threshold vs. Number of loops. Insert: Normalised larger cluster vs. $\theta$ extracted from successive two-dimensional normalised convolution (0 to 100) of random matrix and 2-D disk filter. 
(A)

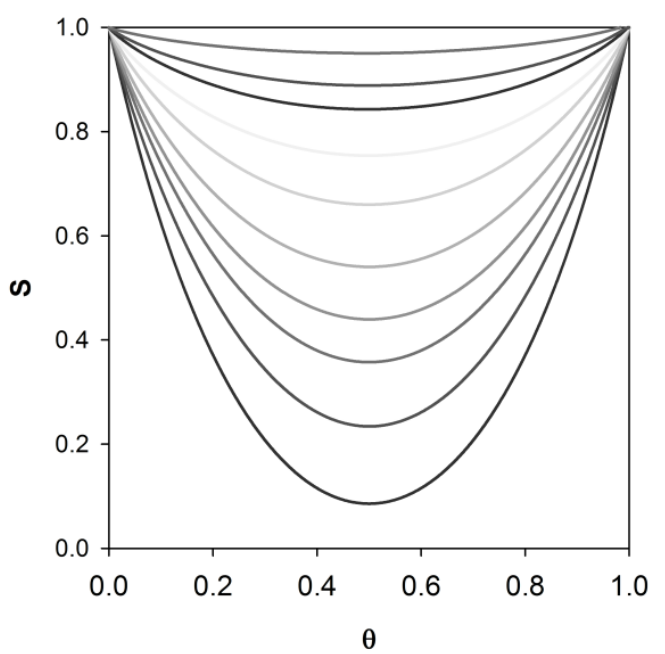

(C)

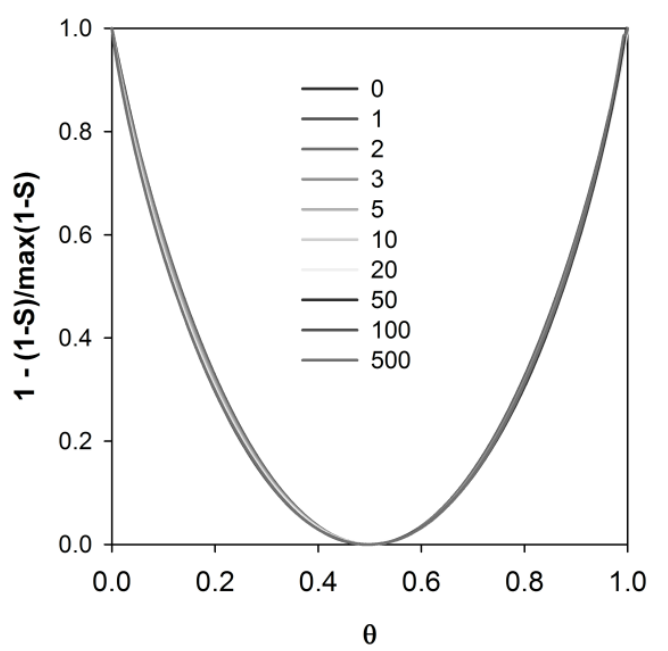

(B)

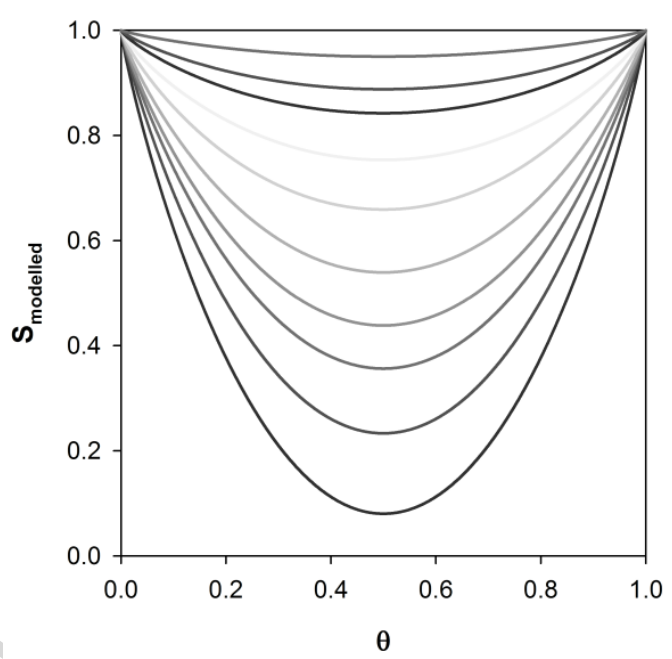

(D)

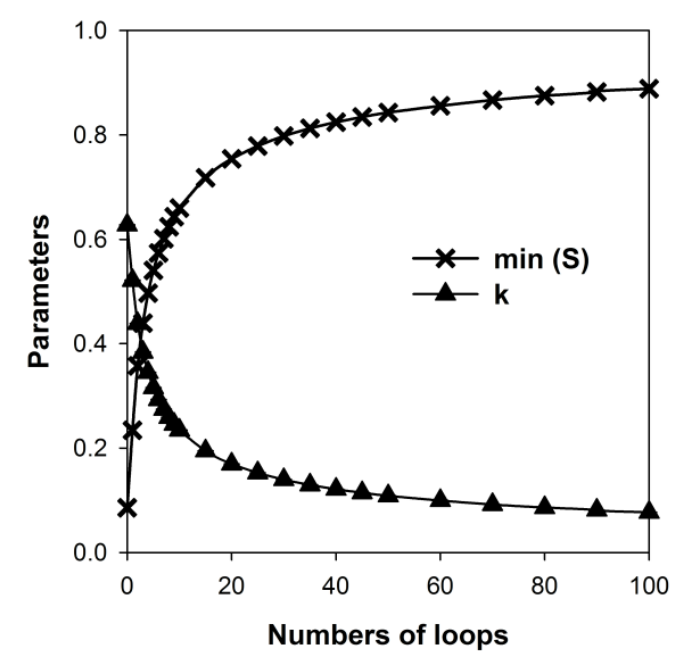

Figure 4:

(A) S vs. $\theta$ extracted from successive two-dimensional normalised convolutions (0 to 500) of random matrix and 2-D disk filter and compared to a random distribution.

(B) Using the Levenberg-Marquardt algorithm, H (i.e. $\mathrm{S}=1-\mathrm{H}$ ) was modelled by the probability density function of the beta distribution $\left(\operatorname{BetaDF}: S(\theta)=1-\mathrm{k} \frac{\theta^{\alpha-1}(1-\theta)^{\beta-1}}{\int_{0}^{1} t^{\alpha-1} \cdot(1-t)^{\beta-1} d t}\right)$ and the best fit (i.e. $S(\theta)=1-k \cdot \frac{\theta(1-\theta)}{1 / 6}$ with $\left.\alpha=\beta=2\right)$ was named $S_{\text {Modelled. }}$

(C) 1-(1-S)/max(1-S) vs. $\theta$ extracted from successive two-dimensional normalised convolutions (0 to 500$)$ of random matrix and 2-D disk filter and compared to a random distribution.

(D) $\{k, \min (S)\}$ vs. Number of loops extracted from figure $4 \mathrm{~A}$ and from the fitting of $S$ vs. $\theta$ and BetaDF function. 


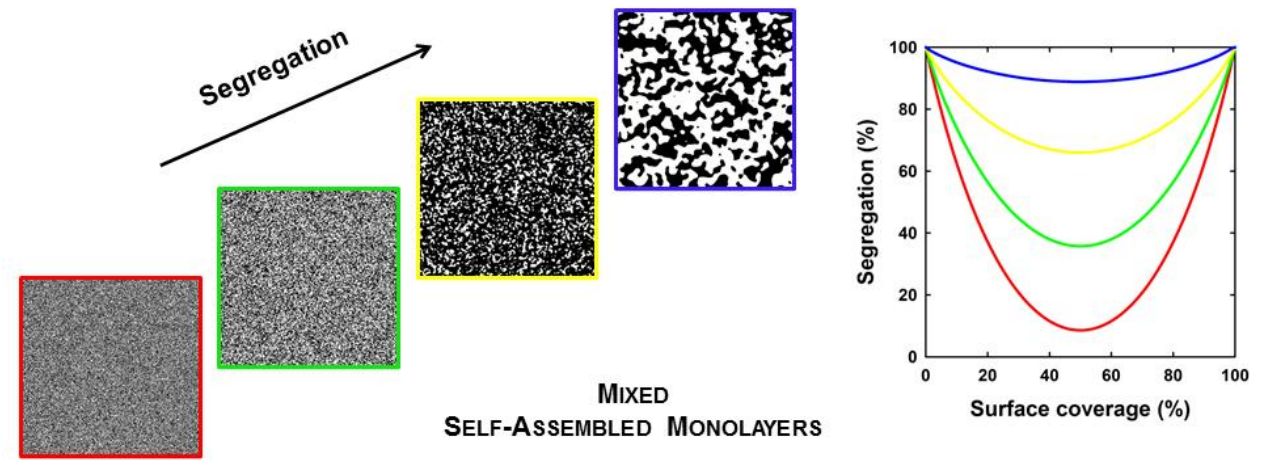

Graphical abstract 
Highlights:

2D segregation on mixed SAM is modelled by image filtering and percolation theory. Local intersite interactions and 2D segregation are linked.

2D segregation persists in a random distribution.

The modelling agrees with experimental data. 